\title{
Engagement für eine innovative Pflege
}

\author{
Der Gemeinschaft dienen und die Qualität garantieren.
}

Täglich setzen sich Pflegepersonen überall in der Welt für innovative Lösungen ein, um bessere Resultate für ihre Patienten zu erreichen. Gleichzeitig helfen sie mit, die allgemeinen Gesundheitskosten zu senken, sei es im Bereich Altenbetreuung, HIV/Aids, Tuberkulose, Armut oder Ressourcenknappheit. Niemals war der Bedarf an neuen Lösungen größer als heute.

Personenbezogene Dienstleistungen nehmen an Bedeutung zu. Zeitlogik und Komplexität der Pflegearbeit und die Beziehung zwischen Pflegenden und Patientinnen sind dabei zu beachten. Wir leben in einer 24-Stunden-Nonstop-Höchstgeschwindigkeitsgesellschaft. Das 24/7 Prinzip, bei dem alles überall verfügbar ist, hat bezüglich Essgewohnheiten und Lebensstil zu Fehlanpassungen zwischen Mensch und Umwelt geführt, was sich offensichtlich an Adipositas, an Stresserkrankungen, an Depressionen, um nur einige der neuen Krankheiten zu nennen, zeigt.

Ich lade Sie ein, mit mir eine Pflegevisite zu unternehmen, den Scheinwerfer auf pflegerische Innovationen zu richten, und zu zeigen, was Pflege tut und kann.

\section{Florence Nightingale}

1) Das Werk von Florence Nightingale ist ein großartiges Beispiel für Innovationen. Eine ihrer vielen Erneuerungen war die Einführung einer systematischen Aufzeichnung. Mitte des 19. Jahrhunderts beobachtete sie, dass Frauen, die zu Hause entbunden hatten, eine geringere Sterblichkeitsrate aufwiesen, als Frauen, die in Spitälern Kinder zur Welt brachten. - Diese systemische Erfassung war die Grundlage Maßnahmen zu setzen, die Müttersterblichkeit zu senken.

\section{Dritte Gesundheitsrevolution}

2) Ilona Kickbusch schreibt in „Die Gesundheitsgesellschaft“: „Wir befinden uns heute in der dritten Gesundheitsrevolution. Die erste sicherte das Überleben, die zweite den Zugang zur medizinischen Versorgung. Heute müssen wir mit der
Entwicklungsdynamik und den Konsequenzen der Gesundheitsgesellschaft zu recht kommen." Wissenschaftler rechneten in einer Studie aus, dass das Fallmanagement bei Personen mit komplexen Krankheiten durch eine Pflegespezialist, die in Abstimmung mit den Ärzten arbeiten, zu potentiellen Einsparmöglichkeiten von 103.000 Dollar pro Jahr je Pflegespezialistin führen könnte.

\section{Känguruhversorgung}

3) Häufig entstehen Innovationen aus Notwendigkeiten heraus, die einen Bedarf oder eine Lücke schließen. Eine amerikanische Pflegekraft hat in Kolumbien die sogenannte Känguruhversorgung als simple, wirtschaftliche, sichere und gesellschaftlich akzeptierte Alternative zur Intensivbehandlung zu früh geborener Säuglinge entwickelt. Dabei werden die gesunden Frühchen mit direktem Hautkontakt zwischen die Brüste ihrer Mütter gelegt. Die Anwendung dieser Methode hat zu einer Senkung der Neugeborenensterblichkeit in Entwicklungsländern geführt. 80 Prozent aller primären Gesundheitspräventionsleistungen werden weltweit von Pflegepersonen geleistet.

\section{Moderne Technologien}

4) Pflegefachkräfte in Südafrika benützen Mobiltelefone um Menschen, die mit HIV/Aids leben, zu unterstützen und um die Einhaltung der Therapieziele zu verbessern. Auf die gleiche Weise gaben Pflegekräfte in Island über das Telefon medizinische Unterstützung und konnten Erschöpfungs- und Verzweiflungssymptome bei Müttern dadurch verringern, dass diese bis zu fünf Telefonanrufe im Zeitraum von zwei Monaten von einer erfahrenen Pflegefachkraft bekamen.

\section{Ambulante Diabetesbetreuung}

5) In den Niederlanden sind innovative Ansätze im Diabetesmanagement unter Leitung von Pflegefachkräften erfolgreich.Diekrankenhausärztliche Versorgung von ambulanten Patienten mit Typ-2-Diabetes wurde von Assistenzärzten auf Pflegespezialisten übertragen und von der ambulanten Klinikversorgung in die allgemein-ärztliche Praxisversorgung verlagert. Dieser Vorgang hatte keine negativen Folgen für die Versorgungsqualität. Im Modellversuch kam es mit dem Einsatz einer Pflegespezialistin sogar zu Verbesserungen bei der Diabetes-Einstellung.

\section{Pflegeexperten}

6) Förderung von Pflegeexperten! Durch forcierte Prophylaxe im Bereich der Druckgeschwüre erfolgte eine Einsparung sowohl im Bereich der Medikamente, Wundtherapeutika, Wundauflagen, als auch bei lokalen Mitteln, wie Wund-, Heilund Fettsalben. Sie brachte eine Kosteneinsparung bei steigender Pflegequalität. Durch den Einsatz eines Experten (freigestellt für diesen Bereich im Pflegezentrum Ybbs mit 180 Personen) konnte innerhalb von sechs Jahren die Dekubitusrate von 14,8 auf 1,1 Prozent gesenkt werden.

\section{Fazit}

Wenn Pflegefachkräfte auch in $\mathrm{Zu}$ kunft mit Mut, Entschlossenheit und Kreativität anpacken, werden Sie weiterhin eine zentrale Bedeutung für die Bewältigung der aktuellen Innovationsprozesse in der Gesundheitsversorgung haben.

Johannes Rieder/GuKPS Mistelbach

\section{LITERATUR}

Hartmut Rosa: Immer schneller, höher, weiter? Wandel - Wendepunkt im System, upgrade 1/09

Das Magazin für Wissen und Weiterbildung er Donau Universität Krems

nternationaler Tag der Pflegenden 2009, Herausgeber: ICN (DBfK, ÖGKV, SBK)

Professionelle Pflege in der GesundheitsverProfessionelle Pflege in der Gesundh
sorgung - wegweisend und innovativ Kickbusch, Ilona: Die Gesundheitsgesell-
Kong - wegweisend und innovativ

schaft - Megatrends der Gesundheit und deren Konsequenzen für Politik und Gesellschaft, Verlag für Gesundheitsförderung, Hamburg 2006 Tewes, Renate: Führungskompetenz ist lernbar, Praxiswissen für Führungskräfte in Gesundheitsberufen, Springer 2009 www.icn.ch/innovations
www.patientenanwaltschaft.com 